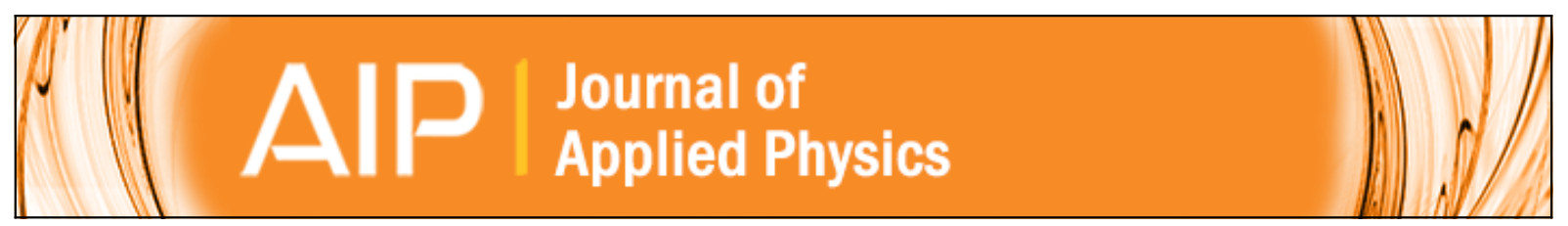

\title{
Derivatization and diffusive motion of molecular fullerenes: Ab initio and atomistic simulations
}

G. Berdiyorov, K. Harrabi, U. Mehmood, F. M. Peeters, N. Tabet, J. Zhang, I. A. Hussein, and M. A. McLachlan

Citation: Journal of Applied Physics 118, 025101 (2015); doi: 10.1063/1.4923352

View online: http://dx.doi.org/10.1063/1.4923352

View Table of Contents: http://scitation.aip.org/content/aip/journal/jap/118/2?ver=pdfcov

Published by the AIP Publishing

\section{Articles you may be interested in}

Atomic packing and diffusion in Fe85Si2B9P4 amorphous alloy analyzed by ab initio molecular dynamics simulation

J. Appl. Phys. 117, 17 B705 (2015); 10.1063/1.4907230

An analysis of hydrated proton diffusion in ab initio molecular dynamics

J. Chem. Phys. 142, 014104 (2015); 10.1063/1.4905077

Single or functionalized fullerenes interacting with heme group

AIP Advances 4, 097119 (2014); 10.1063/1.4895804

Ab initio molecular dynamics simulation of ionic liquids

J. Chem. Phys. 126, 154502 (2007); 10.1063/1.2718531

Band gap engineering in amorphous $\mathrm{Al} \times \mathrm{Ga} 1-\mathrm{x} \mathrm{N}$ : Experiment and ab initio calculations

Appl. Phys. Lett. 77, 1117 (2000); 10.1063/1.1289496

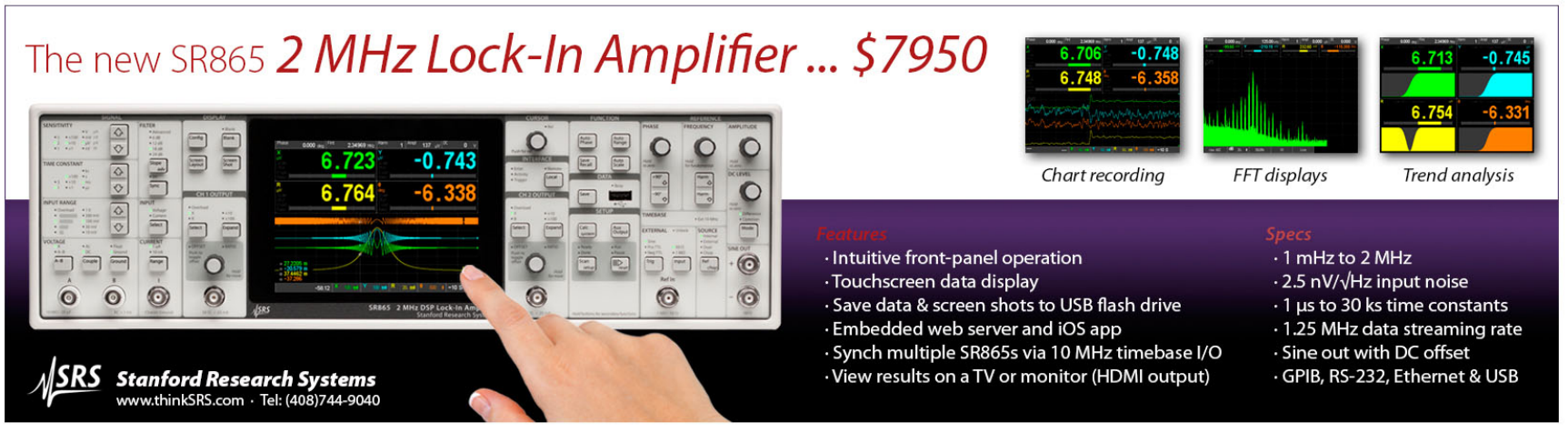




\title{
Derivatization and diffusive motion of molecular fullerenes: $A b$ initio and atomistic simulations
}

\author{
G. Berdiyorov, ${ }^{1, a)}$ K. Harrabi, ${ }^{2}$ U. Mehmood, ${ }^{3}$ F. M. Peeters, ${ }^{4}$ N. Tabet, ${ }^{1}$ J. Zhang, ${ }^{5}$ \\ I. A. Hussein, ${ }^{3}$ and M. A. McLachlan ${ }^{6}$ \\ ${ }^{1}$ Qatar Environment and Energy Research Institute (QEERI), Hamad Ben Khalifa University (HBKU), \\ Qatar Foundation, P.O. Box 5825, Doha, Qatar \\ ${ }^{2}$ Department of Physics, King Fahd University of Petroleum and Minerals, 31261 Dhahran, \\ Kingdom of Saudi Arabia \\ ${ }^{3}$ Department of Chemical Engineering, King Fahd University of Petroleum and Minerals, 31261 Dharan, \\ Kingdom of Saudi Arabia \\ ${ }^{4}$ Departement Fysica, Universiteit Antwerpen, Groenenborgerlaan 171, B-2020 Antwerpen, Belgium \\ ${ }^{5}$ Department of Materials and London Centre for Nanotechnology, Imperial College London, \\ SW7 2AZ London, United Kingdom \\ ${ }^{6}$ Department of Materials and Centre for Plastic Electronics, Imperial College London, \\ SW7 2AZ London, United Kingdom
}

(Received 11 March 2015; accepted 20 June 2015; published online 10 July 2015)

\begin{abstract}
Using first principles density functional theory in combination with the nonequilibrium Green's function formalism, we study the effect of derivatization on the electronic and transport properties of $\mathrm{C}_{60}$ fullerene. As a typical example, we consider [6,6]-phenyl- $\mathrm{C}_{61}$-butyric acid methyl ester (PCBM), which forms one of the most efficient organic photovoltaic materials in combination with electron donating polymers. Extra peaks are observed in the density of states (DOS) due to the formation of new electronic states localized at/near the attached molecule. Despite such peculiar behavior in the DOS of an isolated molecule, derivatization does not have a pronounced effect on the electronic transport properties of the fullerene molecular junctions. Both $\mathrm{C}_{60}$ and PCBM show the same response to finite voltage biasing with new features in the transmission spectrum due to voltage induced delocalization of some electronic states. We also study the diffusive motion of molecular fullerenes in ethanol solvent and inside poly(3-hexylthiophene) lamella using reactive molecular dynamics simulations. We found that the mobility of the fullerene reduces considerably due to derivatization; the diffusion coefficient of $\mathrm{C}_{60}$ is an order of magnitude larger than the one for PCBM. (C) 2015 AIP Publishing LLC. [http://dx.doi.org/10.1063/1.4923352]
\end{abstract}

\section{INTRODUCTION}

Due to their prominent transport properties and electron accepting capability, fullerenes have a great potential for practical applications in organic electronics and photochemistry. ${ }^{1-4}$ One of the notable applications of fullerenes is the fabrication of bulk heterojunction (BHJ) organic photovoltaic cells (OPVs), which consist of positive (p)-type conjugated polymers and n-type fullerene derivatives. The latter provides a promising framework, allowing the formation of a bicontinuous interpenetrating network of electron-donor and acceptor components within the active BHJ layer, which is a crucial factor for achieving high power conversion efficiency. $^{5-7}$ Since such interpenetrating morphology controls many of the performance characteristics of bulk heterojunction organic photovoltaic cells, ${ }^{8-10}$ achieving the morphological stability, particularly under thermal stress conditions, is a key challenge to the commercialization of polymerfullerene OPVs. One of the main reasons for the thermally induced degradation of solar cell performance is the diffusion of fullerene derivatives through the blend film. ${ }^{1-13}$ Although soluble fullerenes have major advances in a $\mathrm{BHJ}$ architecture, the thermal instability of $\mathrm{BHJ}$, due partly to the diffusive motion of molecular fullerenes, must be

\footnotetext{
${ }^{\text {a)} E l e c t r o n i c ~ a d d r e s s: ~ g b e r d i y o r o v @ q f . o r g . q a ~}$
}

addressed before one can consider them for practical applications in OPVs. ${ }^{14}$ Therefore, a fundamental understanding of the diffusive motion of fullerene derivatives within the blend under heat and light pressure is a key factor to increase the lifetime of OPVs. ${ }^{15}$ Here, we implement reactive molecular dynamics (MD) simulations to study the effect of derivatization of fullerenes on their diffusive motion in an ethanol. Derivatization is usually implemented to increase the solubility of fullerenes, thus allowing for solution processing and increase polymer compatibility and miscibility. As an example, we study the dynamics of the fullerene derivative $\mathrm{PC}_{60} \mathrm{BM}$ ([6,6]-phenyl- $\mathrm{C}_{61}$-butyric acid methyl ester), which is called PCBM in what follows, in ethanol solvent. We found that the derivatization reduces the mobility of the fullerene considerably inside the ethanol solvent; the diffusion coefficient is reduced by an order of magnitude due to the attached molecules. Diffusion of PCBM through and its intercalation within the donor polymer domain, formed poly(3-hexylthiophene) (P3HT) polymer is also studied. The mobility of PCBM is smaller as compared to the one for $\mathrm{C}_{60}$ even in this environment. Note that the combination of electron-donating P3HT and electron-accepting PCBM forms one of the most efficient OPV materials. ${ }^{16-20}$

Because of the high electron mobility and affinity, fullerenes have attracted considerable attention as electron 
acceptors and transport materials for organic electronic devices. Since pristine fullerenes are relatively insoluble, and therefore only amenable to thermal evaporation, derivatization is usually applied to increase their solubility. However, such functionalization is known to strongly affect the electronic and transport properties of low dimensional carbon based systems. Therefore, here we use first-principles Density Functional Theory (DFT) calculations in combination with the nonequilibrium Green's function formalism to study the effect of derivatization on the electronic and transport properties of $\mathrm{C}_{60}$ fullerene. As an example, we conduct simulations for a single PCBM molecule with pristine $\mathrm{C}_{60}$ fullerene as a reference. We found that the functionalization has a considerable impact on the electronic properties of the fullerene. For example, extra peaks appear in the density of states (DOS) of the molecule due to the formation of new electronic states localized at/near the attached molecule. However, despite such peculiar features in the DOS of isolated molecules, derivatization does not have a pronounced effect on the electronic transport properties of fullerene molecular junctions, i.e., a fullerene attached to gold wires. Both systems show the same response to external voltage biasing: the transmission decreases with increasing applied voltage, except some resonance peaks in the transmission due to the voltage induced delocalization of some electronic states.

\section{COMPUTATIONAL DETAILS}

The structural and electronic properties of considered systems are studied using DFT calculations ${ }^{21}$ within the generalized gradient approximation (GGA) of Perdew-BurkeErnzerhof (PBE) to represent the exchange-correlation energy. ${ }^{22}$ The samples were first geometry optimized using the convergence criteria for total energy of $10^{-4} \mathrm{eV}$ and Hellman-Feynman forces less than $0.01 \mathrm{eV} / \AA$. The electrostatic potentials were determined on a real-space grid with a mesh cutoff energy of $150 \mathrm{Ry}$ and double-zeta-polarized basis sets of local numerical orbitals were employed for all atoms. Grimme's DFT-D2 empirical dispersion correction ${ }^{23}$ to the PBE was used to account for van der Waals interactions, which is known to be very important for the accurate description of such molecular structures. To study the transport properties of the system, we have constructed a two probe device geometry consisting of $\mathrm{C}_{60}$ and PCBM attached to gold wires, which are subsequently attached to semiinfinite metallic electrodes (see Fig. 2). Quantum transport properties of the system are calculated using the nonequilibrium Green's function formalism with the Brillouin zone sampled with $(4,4,100)$ points within the Monkhorst-Pack $k$-point sampling scheme. All the simulations are conducted using the first-principles computational package Atomistix toolkit (ATK). ${ }^{24}$

In order to study the dynamical properties of fullerene derivatives in a solvent, we conducted MD simulations using reactive force-field ReaxFF, which is a general bond-order dependent potential that provides an accurate description of bond breaking and bond formation during chemical reactions. ${ }^{25}$ The connectivity in the entire system is recalculated in every iteration, enabling dissociation and formation of chemical bonds during the simulations. Non-bounded interactions (van der Waals and Coulomb) are calculated between all atom pairs, irrespective of connectivity, with a short range shielding term. ${ }^{25}$ Since ReaxFF parameters are derived from quantum chemical calculations, such force field gives energies, transition states, reaction pathways, and reactivity trends in agreement with quantum mechanical calculations and experiments. ${ }^{26-28}$ This approach has already been successfully used in describing carbon-based structures. ${ }^{29-31}$ MD simulations are conducted using the computational package LAMMPS. ${ }^{32,33}$

\section{ELECTRONIC TRANSPORT PROPERTIES OF $\mathrm{C}_{60}$ AND PCBM}

We start with considering the electronic properties of isolated $\mathrm{C}_{60}$ and PCBM molecules by calculating the energy dependence of the electron DOS and their molecular energy spectrum. We first conducted structural optimization of both samples using the convergence criterium for energy as $10^{-4}$ $\mathrm{eV}$ between two consecutive steps, and for the maximum force on each atom less than $0.01 \mathrm{eV} / \AA$. The main panel in Fig. 1 shows the DOS of $\mathrm{C}_{60}$ (solid-black curve) and PCBM (dashed-red curve) molecules as a function of electron energy. DOS of the pristine fullerene shows well separated peaks both above and below the Fermi level. Every maximum corresponds to a molecular energy state, as shown in panels 1 and 2 in Fig. 1, where we present the isosurface plots of the highest occupied molecular orbital (HOMO) and the lowest unoccupied molecular orbital (LUMO), respectively (the energies of the states are indicated by verticalgreen lines in the main panel). Both HOMO and LUMO states are characterized by electronic states extended over the entire molecule. They are located at the same energy range away from the Fermi level and the HOMO-LUMO energy gap is $1.64 \mathrm{eV}$.

For the case of PCBM, when we attached the molecule it results in considerable changes in the DOS (dashed-red curve in the main panel of Ref. 1). First, the electron density decreases as compared to one of the pristine fullerenes (compare solid-black and dashed red curves). Second, the HOMO-LUMO gap becomes slightly smaller $(1.47 \mathrm{eV})$. The most pronounced effect of fullerene derivatization is the presence of additional low amplitude peaks in the energy dependence of DOS. These peaks are due to the formation of electronic localized states at/near the attached molecule. Some examples of such delocalized states are shown in panels 5 and 6 , where we plot the projected molecular selfconsistent Hamiltonian (MPSH) eigenstates at energies indicated by $O_{1}$ and $O_{2}$ in the main panel. MPSHs are obtained by projecting the self-consistent Hamiltonian onto the Hilbert space spanned by the basis functions of the central atoms. The eigenstates of the MPSH are associated with the poles of the Green's function and, in general, correspond approximately to the peaks in the transmission spectrum. Thus, the derivatization has a considerable effect on the DOS of fullerenes.

Next, we study the effect of fullerene derivatization on its electronic transport properties and take PCBM as an 

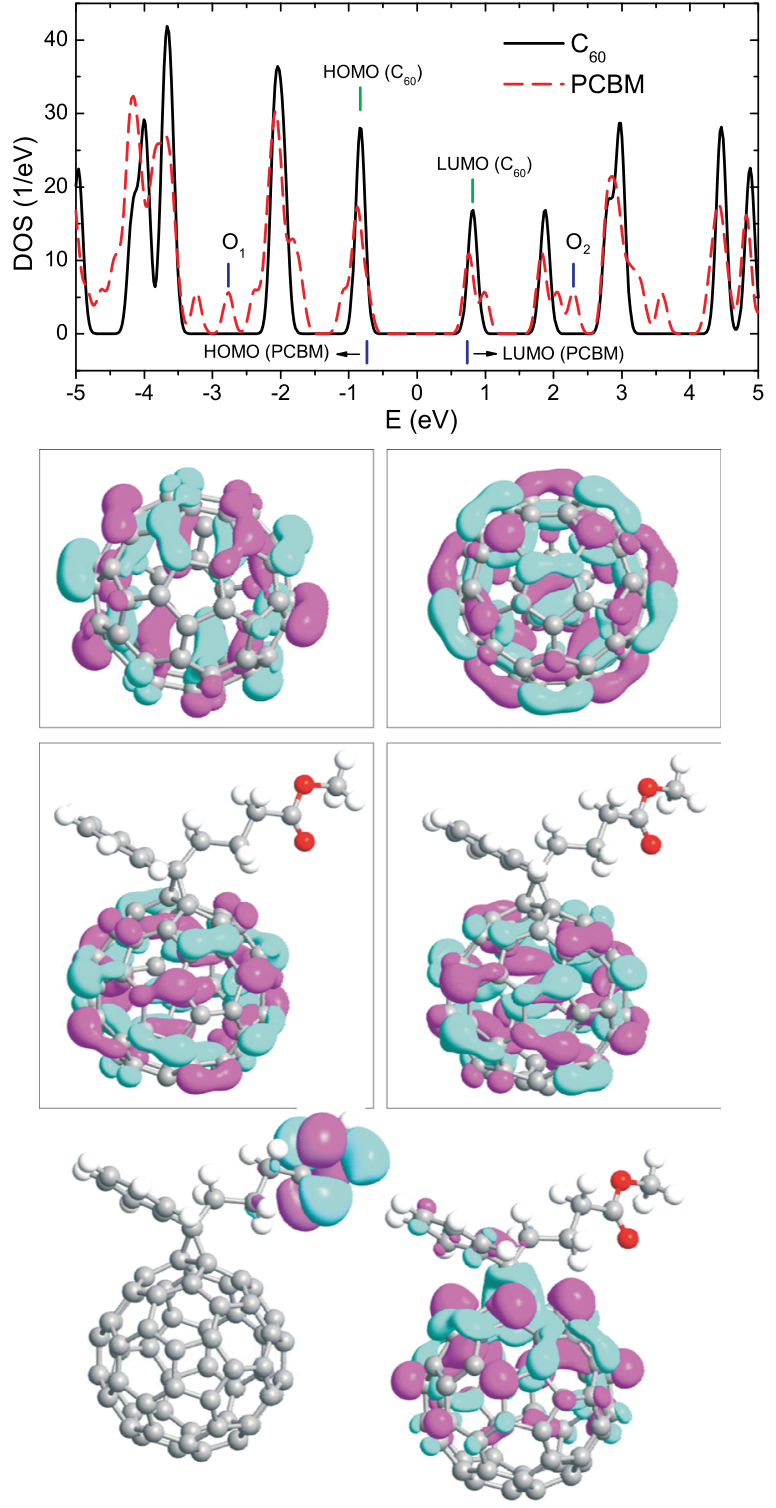

FIG. 1. Main panel: Density of states as a function of energy (the Fermi energy is taken at $E=0$ ) for $\mathrm{C}_{60}$ fullerene (solid-black curve) and PCBM (dashed-red curve). Panels 1-4: Isosurface plots (isovalues of \pm 0.05 ) of the $\operatorname{HOMO}(1,3)$ and LUMO $(2,4)$ orbitals for $\mathrm{C}_{60}(1,2)$ and PCBM $(3,4)$. The energies of the HOMO and LUMO states are indicated by vertical lines in the main panel. Panels 5 and 6 show isosurface plots of the projected selfconsistent hamiltonian eigenstates of the PCBM corresponding to the energies indicated in the main panel by $O_{1}$ and $O_{2}$.

example. For that purpose, we constructed a two probe devise geometry where the optimized PCBM is connected to gold wires through sulfur bonding [see Fig. 2(b)]. Goldsulfur bonding plays an important role in such molecular junctions, resulting in enhanced stability of such nanostructures and increased electronic transmission between gold and sulfur-containing organic molecules (see Ref. 34 for review). The gold wires are consequently connected to metallic electrodes of size $7.064 \AA$, which are modeled as an electron gas with a fixed chemical potential. Similar device structure was also created for pristine $\mathrm{C}_{60}$ fullerene [Fig. 2(a)]. Note that, in both cases we have separated the molecules from their "images" by a distance of at least $10 \AA$.

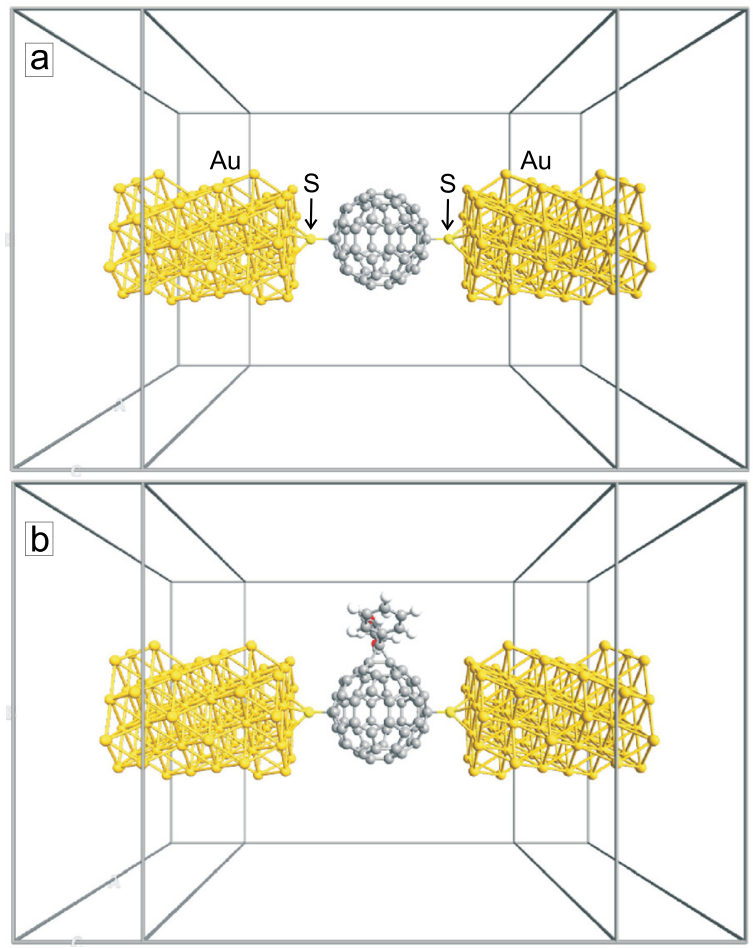

FIG. 2. Molecular device geometry: $C_{60}$ (a) and PCBM (b) connected to gold nanowires through sulfur bonding. The nanowires are connected to metallic electrodes of size $7.064 \AA$.

As a main result, we present in Fig. 3 the zero bias transmission spectra (a), $T(E)$, and device density of states (DDOS) (b) as a function of the electronic energy for $\mathrm{C}_{60}$ (solid-black curves) and PCBM (dashed-red curves). The energy origin is set to be the Fermi level of the system. For both samples, the transmission spectrum is characterized by well separated peaks with different amplitudes both below and above the Fermi level. The amplitude of the peaks is smaller near the Fermi level as compared to the amplitude of the resonance peaks away from the Fermi energy. These features are also reflected in the DDOS of the system as sharp peaks [see Fig. 3(b)], indicating that there is a correspondence between the energy levels of the samples and the transmission spectrum, this is in particular, through the location of their peaks.

At energies away from the Fermi level, the amplitudes of the transmission peaks are almost the same for both samples. However, near the Fermi energy some peaks become larger for PCBM, despite the similar density of states (see Fig. 3). To understand the origin for such local enhancement of the transmission in PCBM, we calculated and compared the transmission eigenstates for different transmission eigenstates at energies corresponding to the resonance peaks in the transmission spectrum. As an example, we show in panels 1 and 2 of Fig. 3 isosurface plots of the transmission eigenstates corresponding to the largest eigenvalue at $E=0.54 \mathrm{eV}$, also indicated on the $T(E)$ curves in Fig. 3(a). It is seen from these plots that the electronic states for $\mathrm{C}_{60}$ are mostly localized near the left electrode with a little extension to the second half of the fullerene (panels 1). However, for the case of PCBM the eigenstates are homogeneous across the sample with larger electron density as compared to $\mathrm{C}_{60}$ 

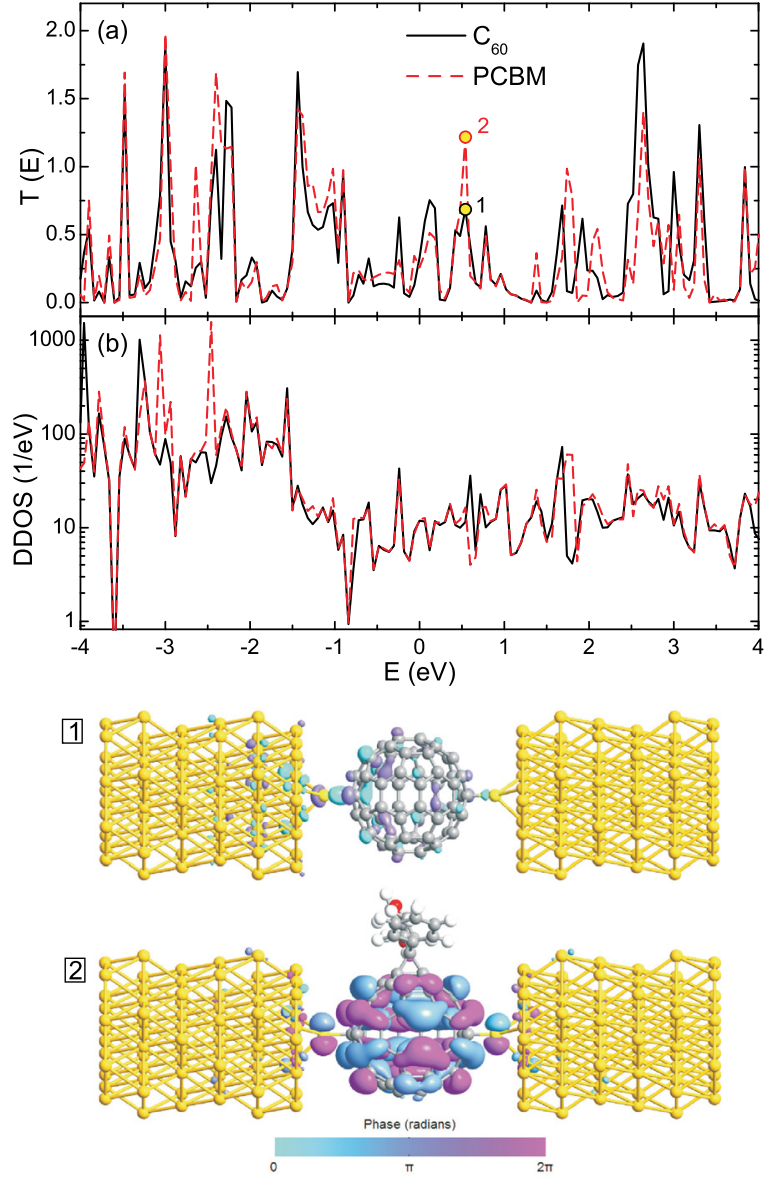

FIG. 3. Zero bias transmission spectrum $T(E)$ (a) and device density of states (b) of $\mathrm{C}_{60}$ (solid-black curves) and PCBM (dashed-red curves) as a function of energy (the Fermi energy is taken at $E=0$ ). Panels 1 and 2: isosurface plots (isovalue 0.15 ) of the transmission eigenstates for the only significant transmission eigenchannel at $k=(0,0)$ and energy $0.54 \mathrm{eV}$, indicated on the $T(E)$ curves. The color map shows the phase of the complex wave functions.

(panel 2). Such delocalization of the states increases the probability of the electrons to cross the system. Thus, derivatization does not have a pronounced effect on the electronic transport in molecular junctions involving fullerenes, except for small features in the transmission spectrum near the Fermi level.

In what follows, we study the response of the considered samples to finite voltage biasing. As a typical example, we present in Fig. 4 the transmission spectra of both $\mathrm{C}_{60}$ (a) and PCBM (b) for two values of the applied voltage: $V=0.5 \mathrm{~V}$ (dashed-red) and $V=1 \mathrm{~V}$ (dotted-blue) (the transmission spectra at zero voltage are presented as a reference by the solid-black curves). We show mostly the region near the Fermi level of the spectra, since for small bias this is the relevant energy range that contributes to carrier transport. It is seen from Fig. 4(a) that the electron transmission spectrum of $\mathrm{C}_{60}$ changes slightly by applying a potential difference across the sample. For example, the transmission is reduced for most of the electron energies except some of the resonance peaks, where the transmission becomes larger at finite biasing. Energy dependent asymmetry in the transmission spectrum is preserved at finite voltage. The PCBM system also shows similar response to voltage biasing [Fig. 4(b)].
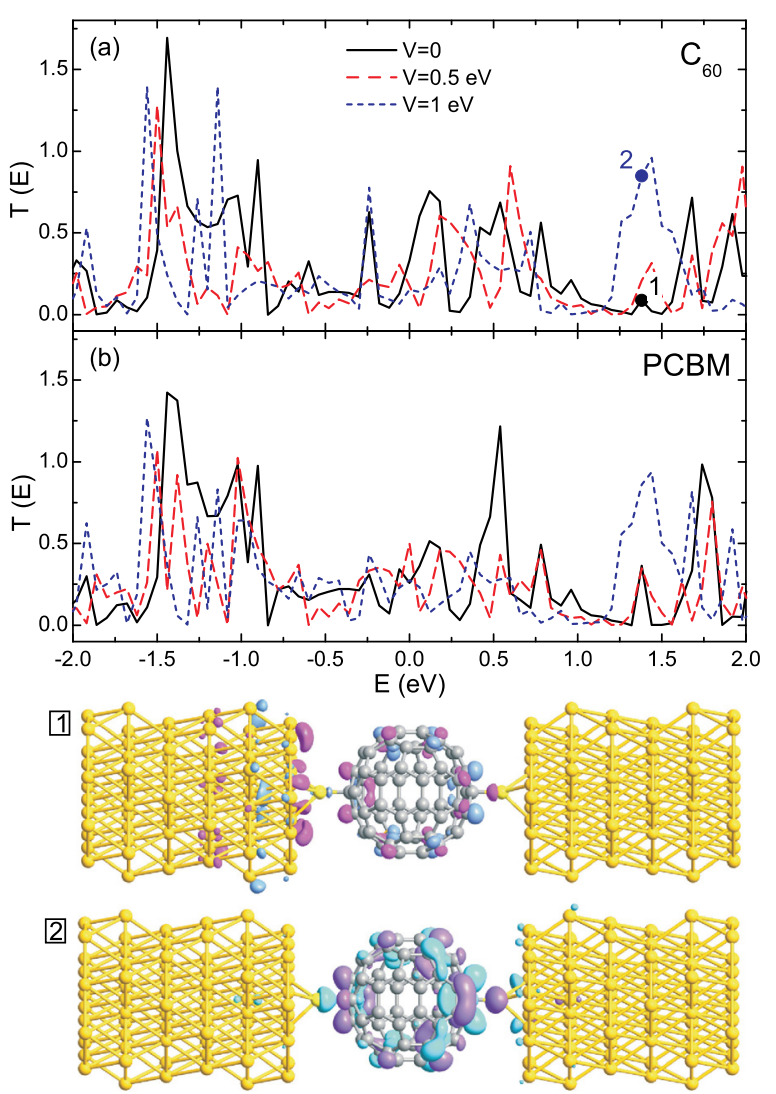

FIG. 4. Transmission spectra of $C_{60}$ (a) and PCBM (b) as a function of energy (the Fermi energy is taken at $E=0$ ) for zero voltage biasing (solidblack curves) and for finite voltage biasing with $V=0.5 \mathrm{~V}$ (dashed-red curves) and $V=1 \mathrm{~V}$ (dotted blue curves). Colored regions highlight the increase of the electronic transmission by applying a voltage difference. Panels 1 and 2: isosurface plots (isovalue 0.15 ) of the transmission eigenstates for the only significant transmission eigenchannel at $k=(0,0)$ and energy $1.38 \mathrm{eV}$, indicated on the $T(E)$ curves. The color map shows the phase of the complex wave functions.

The most prominent feature of the voltage dependence of the transmission for both samples is the increase of the amplitude and broadening of some resonance peaks [see colored regions in Figs. 4(a) and 4(b)]. Calculations for the electron transmission eigenstates show that some states near the transmission peaks become more extended by applying an external voltage (see panels 1 and 2 in Fig. 4), which enhances the electronic transmission through the system. The amplitude of the peaks increases with further increasing the applied voltage, indicating the opening of extra transport channels for the electron. Thus, both considered systems show similar response to the applied voltage with new features in the transmission spectrum.

\section{DYNAMICS OF $\mathrm{C}_{60}$ AND PCBM}

In this section, we study the diffusive motion of $\mathrm{C}_{60}$ and PCBM inside an ethanol solvent and P3HT lamella using classical MD simulations. Our choice of these solvents is based on the facts that ethanol is known as a good general purpose solvent and $\mathrm{P} 3 \mathrm{HT}$ forms one of the promising $\mathrm{BHJ}$ OPVs in combination with PCBM. For our study, we used the ReaxFF force field developed by Monti et al. ${ }^{35}$ To validate this force field for our systems, we have optimized all 

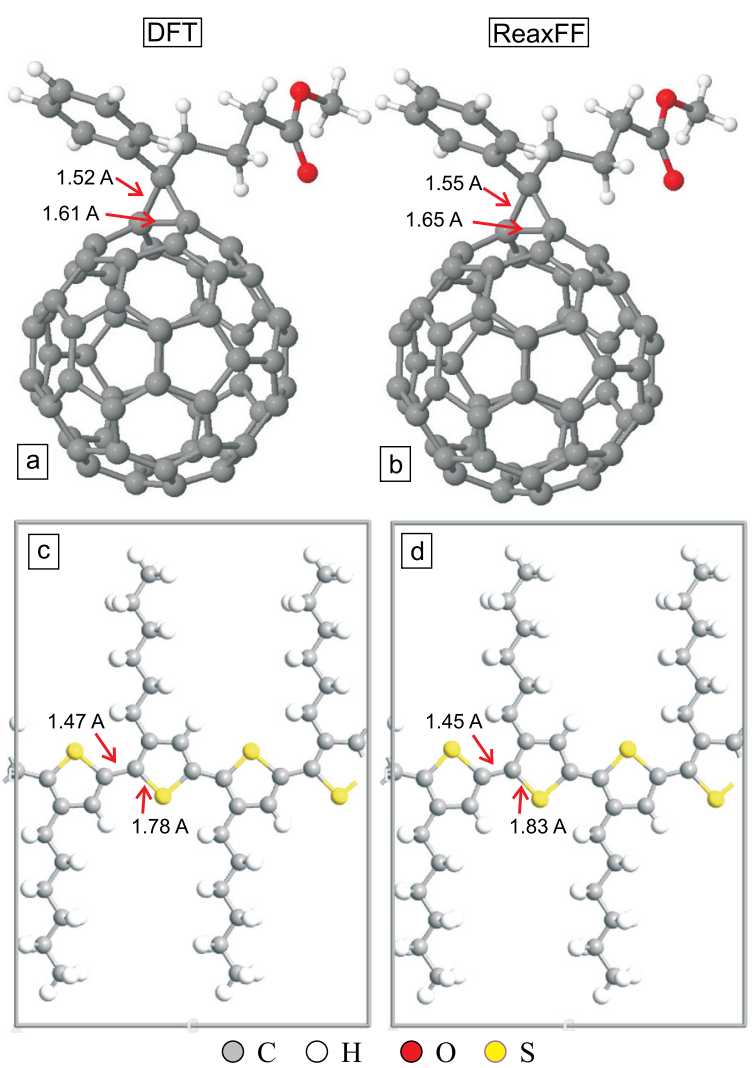

FIG. 5. Optimized structures: equilibrium structures of PCBM (a) and (b) and P3HT (c) and (d) optimized using DFT/PBE (a) and (c) and ReaxFF (b) and (d). Bond distances between selected atoms are shown for comparison.

considered structures using ReaxFF and compared them with the equilibrium structures obtained using DFT/PBE. As an example, we plot in Fig. 5 the optimized structures of PCBM (a) and (b) and P3HT (c) and (d) obtained using DFT/PBE (a) and (c) and ReaxFF (b) and (d), where we highlight some of the bonds. It is seen from this figure that the error in estimating the bond lengths is no more than $0.05 \AA$. Similar results have been observed for $\mathrm{C}_{60}$ and ethanol molecules (not shown here). Using these optimized structures, we constructed a simulation box of size $50 \AA \times 50 \AA \times 50 \AA$ and periodic boundary conditions are implemented in all directions. Studied systems were first energy-optimized at zero temperature and then the temperature of the equilibrated system was ramped up to $500 \mathrm{~K}$ at a rate of $20 \mathrm{~K} / \mathrm{ps}$ using NVT ensemble with a Noose-Hover thermostat for temperature control. When the desired temperature is reached, constant temperature MD simulations were conducted using the NVT ensemble for $600 \mathrm{ps}$. The time step was $0.25 \mathrm{fs}$ and the temperature damping constant was $100 \mathrm{fs}$ in all simulations. For a given temperature, we conducted a statistical analysis and the results presented in this manuscript are averaged over an ensemble of 5 different initial distributions of velocities of the atoms. Here, we present results only for temperature $T=300 \mathrm{~K}$.

We first consider the dynamics of PCBM inside the ethanol solvent. Dynamics of pristine fullerene $\mathrm{C}_{60}$ is also studied for the same conditions as reference. As a main result, we present in Fig. 6(a) the mean square displacement
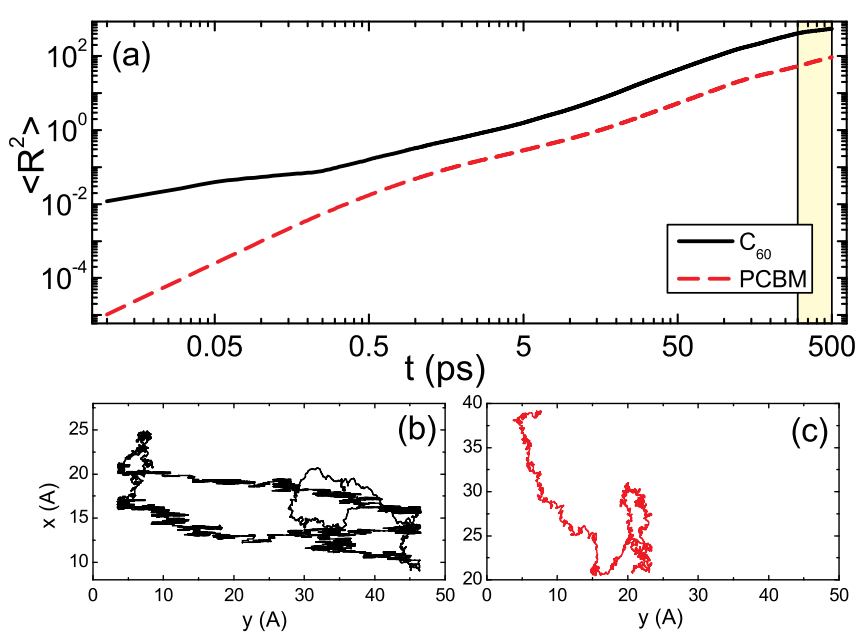

FIG. 6. (a) Mean-square displacement $\left\langle R^{2}\right\rangle\left(\AA^{2}\right)$ as a function of time for the motion of the $\mathrm{C}_{60}$ (solid-black curve) and PCBM (dashed-red curve) molecules at $T=300 \mathrm{~K}$ in ethanol solvent. The region that is used to estimate the diffusion coefficient is highlighted by the yellow area. (b) and (c) $x$-y-trajectories of $\mathrm{C}_{60}$ (b) and PCBM (c) in a simulation box of $50 \AA \times 50 \AA \times 50 \AA$ with periodic boundary conditions in all directions.

$\left\langle R^{2}\right\rangle$ of the $\mathrm{C}_{60}$ (solid-black curve) and PCBM (dashed-red curve) at temperatures $T=300 \mathrm{~K}$ as a function of time, where $R^{2}=x^{2}+y^{2}+z^{2}$. Since the deformation of fullerene is small at these low temperatures, we used the transverse motion of the center of mass of the fullerene ball in constructing the $\left\langle R^{2}\right\rangle(t)$ curves of both systems. It is seen from this figure that the difference in $\left\langle R^{2}\right\rangle$ becomes significant already at small times: the distance that $\mathrm{C}_{60}$ travels in ethanol solvent becomes an order of magnitude larger than the one that is covered by PCBM. This is also clearly seen from Figs. 6(b) and 6(c), where we plot the trajectories of $\mathrm{C}_{60}$ (a) and PCBM (b) during the time of a simulation $500 \mathrm{ps}$ at $300 \mathrm{~K}$. Diffusion coefficients are obtained from the mean square displacement in the large time region [see the yellow region in Fig. 6(a)] as $D=0.166 \AA^{2} / \mathrm{ps}$ and $D=0.034 \AA^{2} / \mathrm{ps}$ for $\mathrm{C}_{60}$ and PCBM, respectively. The found 5 fold decrease of the diffusion coefficient indicates the significance of the derivatization of fullerenes for performance and durability of OPVs.

As we mentioned above, the interpenetrating morphology formed by the electron donor and acceptor materials is critical for the performance of polymer-fullerene BHJ photovoltaic (PV) cells. This also necessitates fullerene derivatization to increase its solubility which is needed to obtain the desired morphology and stability of the active layer. In what follows, we study the dynamics of PCBM and $\mathrm{C}_{60}$ molecules inside P3HT-lamellae, which is considered as one of the promising electron donating polymers in organic solar cell materials. To quantify the mobility of the considered molecules, we show in Fig. 7(a) the mean square displacement $\left\langle R^{2}\right\rangle$ of $\mathrm{C}_{60}$ (solid-black curve) and PCBM (dashed red curve) at $T=300 \mathrm{~K}$. At small times ( $t \leq 100 \mathrm{fs}$ ), both molecules show the same time dependence of $\left\langle R^{2}\right\rangle$. With increasing time, there is a deviation of the curves and the mobility of $\mathrm{C}_{60}$ becomes significantly larger at larger times. The diffusion coefficients of $\mathrm{C}_{60}$ and PCBM are $D=0.018 \AA^{2} / \mathrm{ps}$ and $D=0.001 \AA^{2} / \mathrm{ps}$, respectively, indicating that fullerene 

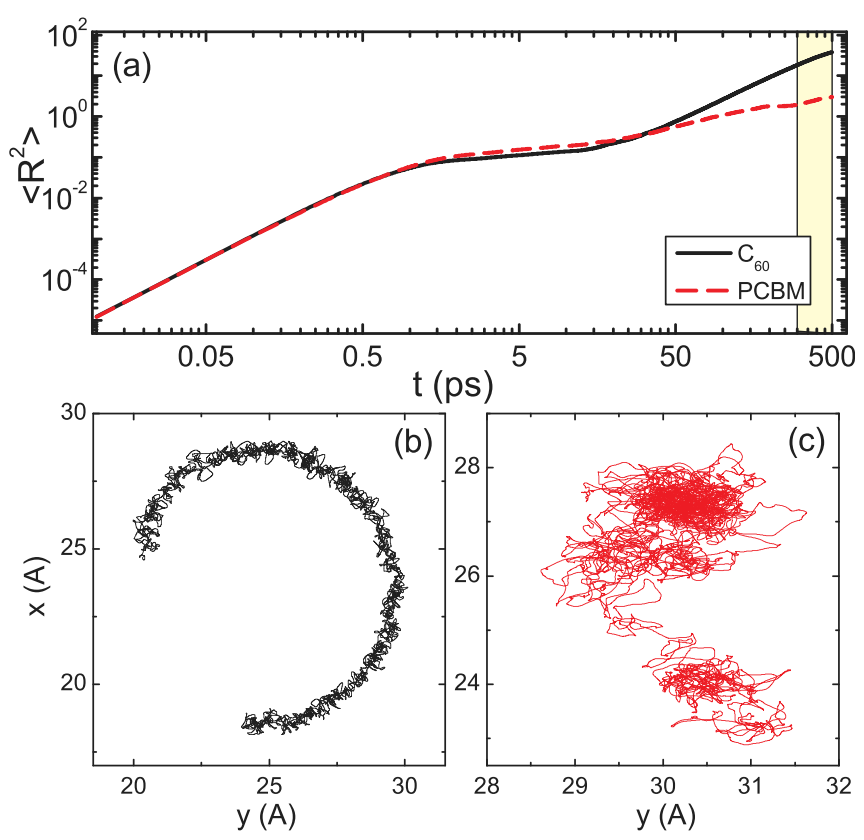

FIG. 7. (a) Mean-square displacement $\left\langle R^{2}\right\rangle\left(\AA^{2}\right)$ as a function of time for the motion of the $\mathrm{C}_{60}$ (solid-black curve) and PCBM (dashed-red curve) molecules at $T=300 \mathrm{~K}$ inside P3HT lamella in a simulation box of $50 \AA \times 50 \AA$ $\times 50 \AA$ with periodic boundary conditions in all directions. The region that is used to estimate the diffusion coefficient is highlighted by the yellow area. (b) and (c) $x$-y-trajectories of $\mathrm{C}_{60}$ (b) and PCBM (c).

functionalization indeed reduces the mobility of fullerenes. Note that the smaller diffusion constants of the fullerenes (fullerene derivatives) inside $\mathrm{P} 3 \mathrm{HT}$ as compared to the ones inside ethanol are due to the larger density of molecules in the former case. Figures 7(b) and 7(b) show the trajectories of $\mathrm{C}_{60}$ and PCBM, respectively, which clearly show the larger diffusion area of the $\mathrm{C}_{60}$ molecule as compared to PCBM. For this solvent, $\mathrm{C}_{60}$ molecule performs mostly circular motion, whereas PCBM does not show such a behavior. The other non-circular trajectories are also obtained during the statistical calculations. Note that the interaction between the molecules and the solvents is purely non-bounded (van der Waals and Coulomb) at the considered temperature. Thus, derivatization considerably reduces the mobility of the fullerenes in the different solvents.

\section{CONCLUSIONS}

First-principles DFT calculations in combination with the Green's functional formalism were used to study the effect of functionalization on the electronic and transport properties of fullerenes. Simulations are conducted for pristine $\mathrm{C}_{60}$ molecule and for its derivative PCBM, which is known to be one of the promising organic compounds to be used in OPVs. Although PCBM shows a richer density of states due to the localized states near/at the attached molecule, similar electronic transport properties have been obtained for the molecular junction made of this molecule as compared to the device made of pristine fullerene. In addition, both systems show similar response to an applied potential difference with new features in the transmission spectrum. However, as our MD simulations revealed, derivatization has a considerable effect on the diffusive motion of fullerenes in different solvents. For example, derivatization can result in a reduction of the diffusion coefficient of fullerenes by an order of magnitude. Our findings can be useful in the understanding of the evolution of the BHJ morphology during the thermal annealing, as well as in understanding the relationship between the morphology of the phase-separated polymer/fullerene blends and the photophysics of the excited states involved in the charge transfer process.

\section{ACKNOWLEDGMENTS}

K.H., U.M. and I.A.H. would like to thank the National Science, Technology and Innovation Program of KACST for funding this research under Project No. 12-ENE2379-04. They also acknowledge support from KFUPM and Research Institute.

${ }^{1}$ H. Imahori, D. M. Guldi, K. Tamaki, Y. Yoshida, C. Luo, Y. Sakata, and S. Fukuzumi, J. Am. Chem. Soc. 123, 6617 (2001).

${ }^{2}$ C. W. Isaacson, M. Kleber, and J. A. Field, Environ. Sci. Technol. 43, 6463 (2009).

${ }^{3}$ S. S. Babu, H. Mohwald, and T. Nakanishi, Chem. Soc. Rev. 39, 4021 (2010).

${ }^{4}$ A. Chuvilin, E. Bichoutskaia, M. C. Gimenez-Lopez, T. W. Chamberlain, G. A. Rance, N. Kuganathan, J. Biskupek, U. Kaiser, and A. N. Khlobystov, Nature Mater. 10, 687 (2011).

${ }^{5}$ G. Yu, J. Gao, J. C. Hummelen, F. Wudl, and A. Heeger, Science 270, 1789 (1995)

${ }^{6}$ R. F. Service, Science 332, 293 (2011).

${ }^{7}$ Z. He, C. Zhong, S. Su, M. Xu, H. Wu, and Y. Cao, Nat. Photonics 6, 591 (2012).

${ }^{8}$ A. Salleo, R. J. Kline, D. M. DeLongchamp, and M. L. Chabinyc, Adv. Mater. 22, 3812 (2010).

${ }^{9}$ B. A. Collins, J. R. Tumbleston, and H. Ade, J. Phys. Chem. Lett. 2, 3135 (2011).

${ }^{10}$ D. M. DeLongchamp, R. J. Kline, and A. Herzing, Energy Environ. Sci. 5, 5980 (2012).

${ }^{11}$ X. Yang, J. K. J. van Duren, R. A. J. Janssen, M. A. J. Michels, and J. Loos, Macromolecules 37, 2151 (2004).

${ }^{12}$ S. Bertho, G. Janssen, T. J. Cleij, B. Conings, W. Moons, A. Gadisa, J. D'Haen, E. Goovaerts, L. Lutsen, J. Manca, and D. Vanderzande, Sol. Energy Mater. Sol. Cells 92, 753 (2008).

${ }^{13}$ S. Ebadian, B. Gholamkhass, S. Shambayati, S. Holdcroft, and P. Servati, Sol. Energy Mater. Sol. Cells 94, 2258 (2010).

${ }^{14}$ Y. Hishikawa, W. Warta, and E. D. Dunlop, Prog. Photovoltaics 20, 12 (2012).

${ }^{15}$ M. Jørgensen, K. Norrman, S. A. Gevorgyan, T. Tromholt, B. Andreasen, and F. C. Krebs, Adv. Mater. 24, 580 (2012).

${ }^{16} \mathrm{G}$. Li, V. Shrotriya, J. Huang, Y. Yao, T. Moriarty, K. Emery, and Y. Yang, Nature Mater. 4, 864 (2005).

${ }^{17}$ Y. Kim, S. Cook, S. M. Tuladhar, S. A. Choulis, J. Nelson, J. R. Durrant, D. D. C. Bradley, M. Giles, I. McCulloch, C.-S. Ha, and M. Ree, Nature Mater. 5, 197 (2006).

${ }^{18}$ M. C. Scharber, D. Mühlbacher, M. Koppe, P. Denk, C. Waldauf, A. J. Heeger, and C. J. Brabec, Adv. Mater. 18, 789 (2006).

${ }^{19}$ G. Dennler, M. C. Scharber, and C. J. Brabec, Adv. Mater. 21, 1323 (2009).

${ }^{20}$ J. Nelson, Mater. Today 14, 462 (2011).

${ }^{21}$ W. Kohn and L. J. Sham, Phys. Rev. 140, 1133 (1965).

${ }^{22}$ J. P. Perdew, K. Burke, and M. Ernzerhof, Phys. Rev. Lett. 77, 3865 (1996).

${ }^{23}$ S. Grimme, J. Comput. Chem. 27, 1787 (2006).

${ }^{24} \mathrm{See} h \mathrm{http} / / / \mathrm{www}$.quantumwise.com for QuantumWise company, Copenhagen, Denmark.

${ }^{25}$ A. C. T. van Duin, S. Dasgupta, F. Lorant, and W. A. Goddard, J. Phys. Chem. A 105, 9396 (2001).

${ }^{26}$ A. Bagri, C. Mattevi, M. Acik, Y. J. Chabal, M. Chhowalla, and V. B. Shenoy, Nat. Chem. 2, 581 (2010). 
${ }^{27}$ F. Castro-Marcano, A. M. Kamat, M. F. Russo, Jr., A. C. T. van Duin, and J. P. Mathews, Combust. Flame 159, 1272 (2012).

${ }^{28}$ K. Chenoweth, S. Cheung, A. C. T. van Duin, W. A. Goddard, and E. M. Kober, J. Am. Chem. Soc. 127, 7192 (2005); K. Chenoweth, A. C. T. van Duin, and W. A. Goddard, J. Phys. Chem. A 112, 1040 (2008).

${ }^{29}$ G. R. Berdiyorov, M. Neek-Amal, F. M. Peeters, and A. C. T. van Duin, Phys. Rev. B 89, 024107 (2014).

${ }^{30}$ G. R. Berdiyorov, M. V. Milošević, F. M. Peeters, and A. C. T. van Duin, Physica B 455, 60 (2014).
${ }^{31}$ A. Sadeghi, M. Neek-Amal, G. R. Berdiyorov, and F. M. Peeters, Phys. Rev. B 91, 014304 (2015).

${ }^{32}$ S. Plimpton, J. Comput. Phys. 117, 1 (1995).

${ }^{33}$ H. M. Aktulga, J. C. Fogarty, S. A. Pandit, and A. Y. Grama, Parallel Comput. 38, 245 (2012).

${ }^{34}$ H. Häkkinen, Nat. Chem. 4, 443 (2012).

${ }^{35}$ S. Monti, A. Corozzi, P. Fristrup, K. L. Joshi, Y. Kyung Shin, P. Oelschlaeger, A. C. T. van Duin, and V. Barone, Phys. Chem. Chem. Phys. 15, 15062 (2013). 